THE KURUME MEDICAL JOURNAL

Vol. 27, p. 5761,1980

\title{
ASPIRIN TREATMENT AND PLATELET FUNCTION IN KAWASAKI DISEASE
}

\author{
TAKASHI YOKOYAMA, HIROHISA KATO, AND EISEI ICHINOSE \\ Department of Pediatrics and Child Health, Kurume University \\ School of Medicine, Kurume, 830, Japan
}

Received for publication January 17, 1980

\begin{abstract}
The comparative effectiveness of small-dose $(30 \mathrm{mg} / \mathrm{kg})$ aspirin and highdose $(150 \mathrm{mg} / \mathrm{kg})$ in Kawasaki disease was evaluated in clinical symptoms and platelet aggregation. Twenty-three patients with Kawasaki disease were entered in the randomized study and devided into three groups. The durations of fever were $14.8 \pm 3.5$ days in the control group, $11.3 \pm 2.1 \mathrm{~d}$. in the small-dose group and $8.7 \pm 2.3 \mathrm{~d}$. in the high-dose group. The high-dose group showed a significantly shorter duration of fever $(\mathrm{P}<.01)$.

The platelet aggregation induced by ADP in the second week of illness were $82.4 \pm 11.6 \%$ in the control group, $66.8 \pm 9.6 \%$ in the small-dose group and $88.0 \pm 10.7 \%$ in the high-dose group $(69.0 \pm 5.0 \%$ in healthy children). The control group and high-dose group showed significantly increased aggregation as compared with healthy children. The platelet aggregation in small-dose group was significantly lower than that in the control group $(\mathrm{P}<.01)$. SGOT and SGPT elevated in half of the patients treated with high dose of aspirin. In Kawasaki disease, $30 \mathrm{mg} / \mathrm{kg} / \mathrm{day}$ of aspirin is recommended to prevent arterial thrombi.
\end{abstract}

\section{INTRODUCTION}

Kawasaki disease (mucocutaneous lymph node syndrome, MCLS) is an acute febrile illness of infants and young children associated with characteristic mucocutaneous involvement and cervical lymphadenopathy. It is estimated that, since it was first reported by Kawasaki in 1967 (Kawasaki et al., 1967), more than 15,000 cases of this disease have occurred in Japan up to 1978. The etiology of this disease remains obscure and no rational treatment measures are available as of today. The most important clinical problem is sudden death from coronary arteritis accompanied by aneurysms and thrombotic occlusion (Kato et al.,
1975). The approximate mortality rate is $1.4 \%$. Our previous study, however, has demonstrated that aspirin was effective on coronary lesions in Kawasaki disease (Kato et al., 1979). In addition, $30 \mathrm{mg} / \mathrm{kg}$ dose of aspirin showed a suppressing effect on the hyperaggregability of platelet in this disease, and it has been considered to be useful to prevent the formation of arterial thrombi (Yokoyama et al., 1977). In this study, we attempted to use high doses of aspirin such as that used in collagen vascular disease, expecting its antiinflammatory effects. The comparative study on the effectiveness of small or high doses of aspirin in Kawasaki disease was evaluated in clinical symptoms and platelet aggregation. 


\section{PATIENTS AND METHODS}

Twenty-three patients diagnosed as Kawasaki disease according to the guidelines (Table 1) at Kurume University Hospital were entered in the randomized study of small-dose versus high-dose aspirin therapy. The age of these patients ranged from 3 months to 6 years and there were 14 males and 9 females. They were devided into three groups treated with three different regimens ; 7 patients were treated with antibiotic $(50 \mathrm{mg} / \mathrm{kg} /$ day of cephalexine) only for one week (control group), 30 $\mathrm{mg} / \mathrm{kg}$ dose of aspirin was administered to 9 patients daily until coronary angiography was performed (small-dose group) and $100-150 \mathrm{mg} / \mathrm{kg}$ dose of aspirin was administered to 7 patients until serum salicylate levels reached $25 \mathrm{mg} / 100 \mathrm{ml}$ and/or the appearance of

TABLE 1

Diagnostic guideline of Kawasaki disease (August 1978)

\section{A. PRINCIPAL SYMPTOMS}

1. Fever of unknown etiology lasting 5 days or more

2. Bilateral congestion of ocular conjunctivae

3. Changes of lips and oral cavity

(1) Dryness, redness and fissuring of lips

(2) Protuberance of tongue papillae (strawberry tongue)

(3) Diffuse reddening of oral and pharyngeal mucosa

4. Changes of peripheral extremities

(1) Reddening of palms and soles (initial stage)

(2) Indurative edema (initial stage)

(3) Membranous desquamation from fingertips (convalescent stage)

5. Polymorphous exanthema of body trunk without vesicles or crusts

6 . Acute nonpurulent swelling of cervical lymphnodes of $1.5 \mathrm{~cm}$ or more in diameter At least five items of $1 \sim 6$ should be satisfied for diagnosis of MCLS.

\section{B. OTHER SIGNIFICANT SYMPTOMS OR FINDINGS}

1. Carditis, especially myocarditis and pericarditis

2. Diarrhea

3. Arthralgia or arthritis

4. Proteinuria and increase of leukocytes in urine sediment

5. Changes in blood tests

(1) Leukocytosis with shift to the left

(2) Slight decrease in erythrocyte and hemoglobin levels

(3) Increased ESR (4) Positive CRP (5) Increased $\alpha_{2}$-globulin

(6) Thrombocytosis (7) Negative ASO

6. Changes occasionally observed

(1) Aseptic meningitis

(2) Mild jaundice or slight increase of serum transaminase

(3) Swelling of gall bladder

Remarks: 1. Male Female ratio: $1.5 / 1$, patients under 5 years of age $: 80 \%$, fatality rate: $1 \sim 2 \%$ (usually sudden death due to coronary thrombosis), recurrence rate $: 2 \sim 3 \%$.

2. Complication after recovery: occasionally myocardial infarction and mitral insufficiency.

3. Autopsy: coronary aneurysm, thrombosis and myocarditis in most cases. 
any sign of salicylate intoxication. The platelet count and platelet aggregation were determined every week in all cases. In some cases, serum salicylate levels at two hours after ingestion of aspirin were determined every three days by Keller's method (Keller, 1947). The number of platelets was counted by Brecher-Cronkite technique. Platelet aggregation was estimated from changes in optical transmission of citrated platelet-rich plasma at $37^{\circ} \mathrm{C}$ with a concentration of $400,000 / \mathrm{mm}^{3}$ using Bryston aggregometer. As an inducer of aggregation, ADP (final concentration, $4.8 \times 10^{-6} \mathrm{~mol}$ ) was used. The de-
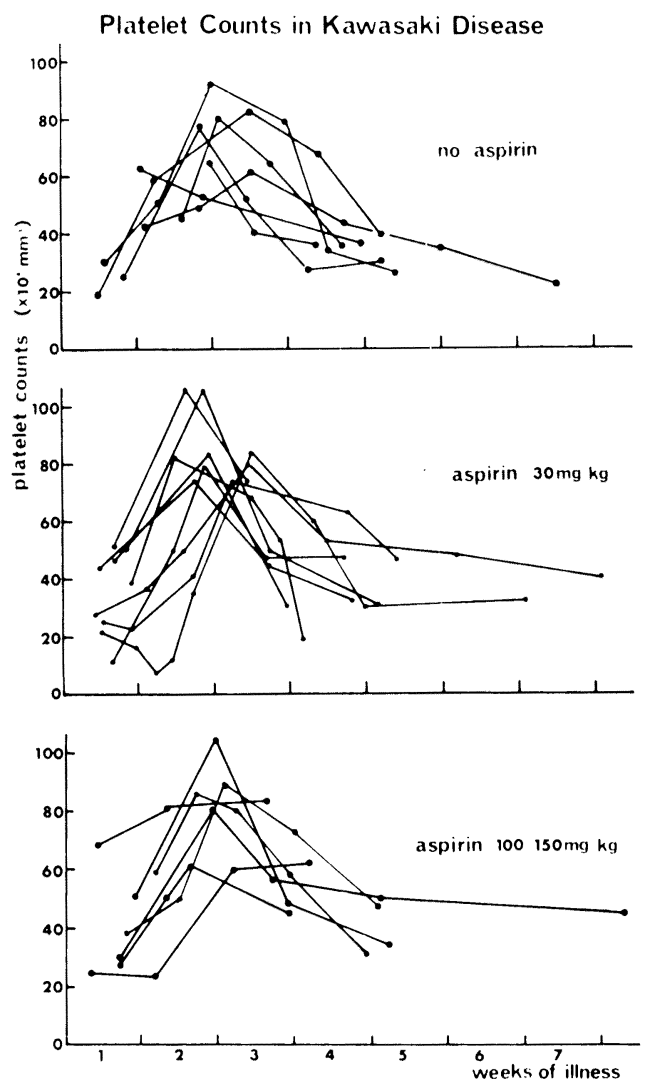

Fig. 1. Chages in platelet counts in patients with Kawasaki disease. gree of platelet aggregation was evaluated as the rate of maximal aggregation regardless of time.

\section{RESULTS}

Platelet counts in the second or third week of illness were elevated in all cases (Fig. 1). The maximum value ranged from $580,000 / \mathrm{mm}^{3}$ to $1,140,000 /$ $\mathrm{mm}^{3}\left(809,000 \pm 142,000 / \mathrm{mm}^{3}\right)$. The number of platelets started increasing in the second week of illness, reached a peak at around the third week and returned to a normal range at the fifth week of illness. There was no difference between the control group and groups treated by small dose or high dose aspirin. Platelet aggregation was accelerated from the second week to the fourth week of illness in the control group, and its average value was $82.4 \pm 11.6 \%$ in the second week of illness (Fig. 2). The platelet aggregation was significantly increased compared with that in healthy children $(69.0 \pm 5.0 \%)$. In the small-dose group, the average value of platelet aggregation was $66.8 \pm 9.6 \%$ in the second week of illness and $64.7 \pm 7.1 \%$ in the third week of illness (Fig. 2). These values were significatly lower than that of the control group $(\mathrm{P}<.01)$. Meanwhile, in the high-dose group, the average value of the platelet aggregation was $88.0 \pm 10.7 \%$ in the second week of illness. There was no significant difference between the high-dose group and the control group. In seven patients given $150 \mathrm{mg} / \mathrm{kg}$ dose of aspirin, serum salicylate lavels were low, ranged from $1.9 \mathrm{mg} / 100 \mathrm{ml}$ to $16.0 \mathrm{mg} / 100 \mathrm{ml}(9.3 \pm 4.6$ $\mathrm{mg} / 100 \mathrm{ml}$ ) in the $7 \mathrm{th}$ day of the illness. However, at the third or fourth week of the illness when acute manifestiations disappeared, serum salicylate levels increased significantly and reached to $40.0 \mathrm{mg} / 100 \mathrm{ml}$ in one case. 
Platelet Aggregation in Kawasaki Disease

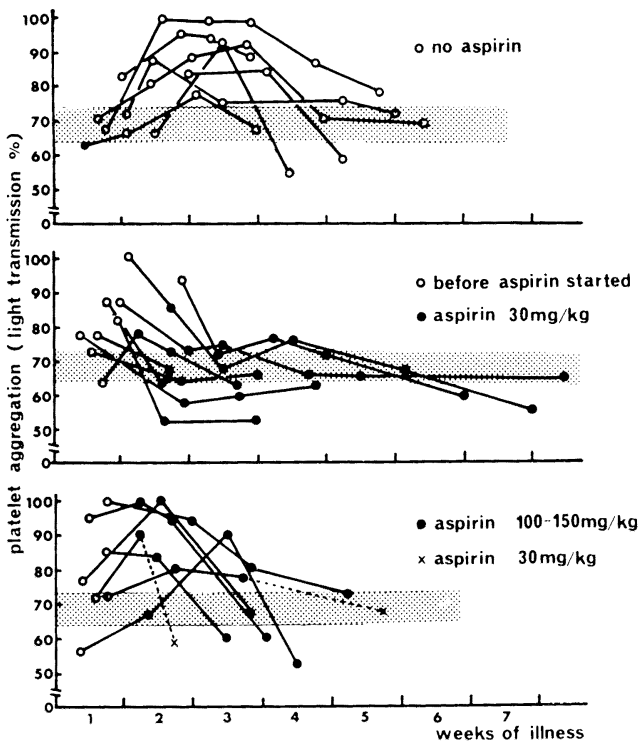

Fig. 2. ADP-induced piatelet aggregation in patients with Kawasaki disease. Stippled area indicates platelet aggregation in healthy children (mean $\pm \mathrm{S}$. D.).

The average duration of fever (above $37^{\circ} \mathrm{C}$ ) was $14.8 \pm 3.5$ day in the control group, $11.3 \pm 2.1$ day in the small-dose group and $8.7 \pm 2.3$ days in the highdose group. The high-dose group showed a sighificantly shorter duration of fever $(\mathrm{P}<.01) .1 / 7$ cases $(14 \%)$ in the control group, $8 / 16$ cases $(50 \%)$ in the small-dose group and $2 / 8$ cases (25\%) in the high-dose group had improved in erythrocyte sedimentation rate by the third week of the illness. There was no significant difference between the small-dose group and the high-dose group.

\section{DISCUSSION}

Thrombocytosis in Kawasaki disease was first reported by Asai and cowor- kers (Asai et al., 1973). The mechanism of thrombocytosis and platelet hyperaggregability in Kawasaki disease remains elusive. This thrombocytosis and hyperaggregability may be caused by the association with angitis and/or the presence of the immune-complex. In our investigation, $27 / 150$ cases (17\%) showed coronary aneurysms on coronary angiography (Kato et al., 1979): in these cases, thrombocytosis and hyperaggregability could lead to formation of arterial thrombi. It is recognized that platelet aggregation plays a major role in initiation of an arterial thrombus (Weiss, 1975). According to our results, platelet aggregation was suppressed in the small-dose group; on the other hand, it was not suppressed in the high-dose group. This fact may be explained by the hypothesis that a small dose of aspirin inhibits the generation of thromboxan $\mathrm{A}_{2}$ which is inducer of platelet aggregation, and furthermore, a high dose of aspirin inhibits vessel wall production of $\mathrm{PGI}_{2}$ (prostacyclin) as well, which is an inhibitor of platelet aggregation (Kelton et al., 1978). A high dose of aspirin was given to seven patients, expecting its anti-inflammatory effect as well as antithrombotic effect. It is accepted that serum salicylate levels of 20 to $30 \mathrm{mg} / 100 \mathrm{ml}$ are required for the optimal anti-inflammatory effect in patients with rheumatic disease. However, serum salicylate levels were markedly lower, contrary to our expectation in the acute phase. But when acute manifestiation disappaared, serum salicylate levels had begun to rise rapidly and reached $40 \mathrm{mg} /$ $100 \mathrm{ml}$ in one case. This suggests that Kawasaki disease patients had malabsorption of aspirin during the acute phase, which was already reported by Jacobs (Jacobs, 1978). The high-dose group revealed a significantly shorter duration of fever as compared with the 
small-dose and control group $(\mathrm{P}<.01)$. However, elevated ESR and positive Creactive protein did not return to normal even after the disappearance of fever, which was not significant compared with the small-dose group. The ultimate purpose in the treatment of Kawasaki disease is not to shorter the duration of fever, but to reduce coronary abnormalities and to prevent the formation of arterial thrombi. Although high doses of aspirin have some effects on fever in Kawasaki disease, it is rather detrimental in preventing arterial thrombi because of its thrombogenic effect (Kelton et al., 1978). In addition, careful monitoring of patients treated with high doses of aspirin is necessary to predict salicylate intoxication, and SGOT and SGPT elevated in the half of those patients treated with high dose aspirin who we observed. However, further evaluation by coronary angiography should be necessary. In conclusion, the authors recommend that aspirin be administered in Kawasaki disease in doses of $30 \mathrm{mg}$ / $\mathrm{kg}$ per day.

\section{REFERENCES}

Asal, T., Kiguchi, H., Nagai, Y. and Kusakawa, S. (1973). Analysis of cardiac involvement in 29 cases with M. C. L. S. (in Japanese).
Japanese Journal of Pediatrics, 26, 824-830.

JACOBS, J. C. (1978). Successful treatment of Kawasaki disease with high-dose aspirin. Pediatric Research (abstracts). 12, 494.

Keller, W. J. (1947). A rapid method for the determination of salicylates in serum or plasma. Am. J. Clin. Path., 17, 415-417.

Kelton, J. G., Hirsh, J., Carter, C. J. and Buchanan, M. R. (1978). Thrombogenic effect of high-dose aspirin in rabbits. J. Clin. Invest. 62, 892-895.

Kato, H., Kolke, S., Yamamoto, M., Ito, Y. and YANO, E. (1975). Coronary aneurysms in infants and young children with acute febrile mucocutaneous lymph node syndrome. J. Pediatr., 86, 892-898.

Kato, H., Koike, S. and Yokoyama,T. (1979). Kawasaki disease: Effect of treatment on coronary artery involvement. Pediatrics, 63, $175-179$.

KAWASAKI, T. (1967). Acute febrile mucocutaneous syndrome with lymphoid involvement with specific desquamation of the fingers and toes in children. Jap. J. Allerg., 16, 178-222.

Kawasaki, T., Kosaki, F., Okawa, S., ShigemaTSU, I. and YanaGawa, H. (1974). A new infantile acute febrile mucocutaneous lymph node syndrome (MCLS) prevailing in Japan. Pediatrics, 54, 271-276.

Weiss, H. J. (1975). Platelet physiology and abnormalities of platelet function (First of two parts). N. Engl. J. Med., 293, 531-541. Yokoyama, T., Kato, H. and Kolke, S. (1977), Platelets and aspirin in Kawasaki disease. Presented at the MCLS Research Committee Meeting, Tokyo, February 1977. 